\title{
Local stimulation of articular cartilage repair by transplantation of encapsulated chondrocytes overexpressing human fibroblast growth factor 2 (FGF-2) in vivo ${ }^{\dagger}$
}

\author{
Gunter Kaul ${ }^{1}$ \\ Magali Cucchiarini $^{1}$ \\ David Arntzen ${ }^{1}$ \\ David Zurakowski ${ }^{2}$ \\ Michael D. Menger ${ }^{3}$ \\ Dieter Kohn ${ }^{1}$ \\ Stephen B Trippel ${ }^{4}$ \\ Henning Madry ${ }^{1 *}$ \\ ${ }^{1}$ Laboratory for Experimental
Orthopaedics, Department of
Orthopaedic Surgery, Saarland
University Medical Center, Homburg,
Germany \\ ${ }^{2}$ Departments of Biostatistics and \\ Orthopaedic Surgery, Children's \\ Hospital, Harvard Medical School, \\ Boston, Massachusetts, USA \\ ${ }^{3}$ Institute for Clinical and \\ Experimental Surgery, Saarland \\ University Medical Center, Homburg, \\ Germany
}

${ }^{4}$ Department of Orthopaedic Surgery, Indiana University, Indianapolis, Indiana, USA

*Correspondence to:

Henning Madry, Laboratory for Experimental Orthopaedics,

Department of Orthopaedic Surgery, Saarland University Medical Center, 66421 Homburg, Germany.

E-mail: hmad@hotmail.com

${ }^{\dagger}$ Part of this work was presented at the 51st Annual Meeting of the Orthopaedic Research Society, February 20-23, 2005, Washington, DC, USA.

Received: 19 January 2005

Revised: 13 May 2005

Accepted: 13 May 2005

\begin{abstract}
Background Defects of articular cartilage are an unsolved problem in orthopaedics. In the present study, we tested the hypothesis that gene transfer of human fibroblast growth factor 2 (FGF-2) via transplantation of encapsulated genetically modified articular chondrocytes stimulates chondrogenesis in cartilage defects in vivo.
\end{abstract}

Methods Lapine articular chondrocytes overexpressing a lacZ or a human FGF-2 gene sequence were encapsulated in alginate and further characterized. The resulting lacZ or FGF-2 spheres were applied to cartilage defects in the knee joints of rabbits. In vivo, cartilage repair was assessed qualitatively and quantitatively at 3 and 14 weeks after implantation.

Results In vitro, bioactive FGF-2 was secreted, leading to a significant increase in the cell numbers in FGF-2 spheres. In vivo, FGF-2 continued to be expressed for at least 3 weeks without leading to differences in FGF-2 concentrations in the synovial fluid between treatment groups. Histological analysis revealed no adverse pathologic effects on the synovial membrane at any time point. FGF-2 gene transfer enhanced type II collagen expression and individual parameters of chondrogenesis, such as the cell morphology and architecture of the new tissue. Overall articular cartilage repair was significantly improved at both time points in vivo.

Conclusions The data suggest that localized overexpression of FGF-2 enhances the repair of cartilage defects via stimulation of chondrogenesis, without adverse effects on the synovial membrane. These results may lead to the development of safe gene-based therapies for human articular cartilage defects. Copyright (c) 2005 John Wiley \& Sons, Ltd.

Keywords cartilage defects; FGF-2; transfection; chondrocytes; alginate; transplantation

\section{Introduction}

Adult articular cartilage has a very limited capacity for repair [1,2]. Cartilage defects that penetrate the underlying subchondral bone, such as resulting from direct trauma, are initially repopulated with mesenchymal cells from the bone marrow [3]. Over time, many of these pluripotent cells differentiate into chondrocytes, and deposit a cartilaginous matrix [3]. The repair tissue 
that results from that chondrogenesis has inferior structural characteristics and degenerates over the course of some months [2]. If let untreated, such cartilaginous lesions may further progress and eventually lead to osteoarthritis of the affected joint. An imbalance of polypeptide growth factors acting upon the cells that initially repopulate the defect is thought to play an important role in these events [4]. Consequently, chondrogenesis might be enhanced by regenerative signals provided to the site of articular cartilage repair.

Fibroblast growth factor 2 (FGF-2), a member of the multifunctional fibroblast growth factor family $[5,6]$, is mitogenic for articular chondrocytes [7,8]. In vivo, FGF-2 stimulates the differentiation of mesenchymal cells during chondrogenesis [9,10] and has been reported to improve the repair of cartilage defects [11-16]. However, FGF-2 protein has a very short plasma half-life [14,17] and is rapidly cleared from the synovial fluid [18]. To achieve a physiological effect of the protein, either high doses $[11,13]$ or continuous delivery via osmotic pumps $[14,15]$ are required. Recent investigations have suggested that gene transfer may be an alternative to directly produce therapeutic factors at sites of articular cartilage damage [19,20]. Transgenes have been expressed in cartilage defects either by transplantation of chondrocytes genetically modified using nonviral [21] or viral vectors [22-25], or by direct vector application [26]. When alginate spheres carrying transfected chondrocytes were transplanted into articular cartilage defects, transgene expression persisted for at least 32 days in vivo [21]. Nonviral, lipid-mediated overexpression of human FGF-2 was already shown to selectively stimulate cell proliferation in a model of articular chondrocyte transplantation in vitro [27]. However, it remains unknown if bioactive FGF-2 can be secreted from alginate spheres containing transfected chondrocytes. It also remains unknown if transplantation of these spheres to sites of articular cartilage damage would modulate the chondrogenesis and cartilage repair in vivo in a safe manner. In the present study, we tested the hypothesis that lipid-mediated overexpression of human FGF-2 via transplantation of alginate spheres containing genetically modified articular chondrocytes enhances chondrogenesis and stimulates articular cartilage repair in vivo. To determine the effects of FGF-2 overexpression at the beginning of chondrogenesis [3], a first time point of 3 weeks after transplantation was chosen for evaluation. Since the repair tissue begins to degenerate by 12 weeks in this model system [3], a second time point of 14 weeks was selected to evaluate the long-term effects of FGF-2 overexpression on articular cartilage repair.

\section{Materials and methods}

\section{Materials}

Plasticware was obtained from Falcon (Becton Dickinson, Pont de Claix, France). All reagents were from Invitrogen/GIBCO (Karlsruhe, Germany) unless otherwise noted. Bovine testicular hyaluronidase, Lcystein, $\mathrm{Na}_{2}$ ETDA, calf thymus DNA and alginate were from Sigma (Munich, Germany). Collagenase type I (activity: $232 \mathrm{U} / \mathrm{mg}$ ) was from Biochrom (Berlin, Germany). Dimethylmethylene blue was from Serva (Darmstadt, Germany). Chondroitin-6-sulfate from shark cartilage was purchased from Fluka (Buchs, Switzerland).

\section{Cell culture and lipid-based transfection}

Articular cartilage was harvested from the knee and hip joints of three male Chinchilla bastard rabbits (mean weight: $1.9 \pm 0.2 \mathrm{~kg}$; Charles River, Sulzfeld, Germany). The cartilage was washed twice with phosphate-buffered saline (PBS), diced into $2.0 \times 2.0 \times 0.5 \mathrm{~mm}$ fragments, and digested in spinner bottles containing Dulbecco's modified Eagle's medium (DMEM) with $50 \mu \mathrm{g} / \mathrm{ml}$ ascorbic acid, $100 \mathrm{U} / \mathrm{ml}$ penicillin $\mathrm{G}, 100 \mu \mathrm{l} / \mathrm{ml}$ streptomycin (basal medium) and $0.02 \%$ collagenase in a humidified atmosphere with $10 \% \mathrm{CO}_{2}$ at $37^{\circ} \mathrm{C}$ for $16 \mathrm{~h}$. Isolated cells were filtered through a $125 \mu \mathrm{m}$ mesh to remove undigested matrix. Viability was determined by trypan blue exclusion and always exceeded $90 \%$. The cell number was determined by hemocytometry.

Isolated chondrocytes were placed in monolayer culture in $100-\mathrm{mm}$ dishes in basal medium containing $10 \%$ fetal bovine serum (growth medium). Transfections were performed with subconfluent cells, 10-14 days after cell isolation, using endotoxin-free expression plasmid vectors carrying either the E. coli lacZ gene (pCMVlacZ; termed lacZ-transfected), or a human FGF2 cDNA (pCMVhFGF-2; termed FGF-2-transfected) [27] placed under the control of the human cytomegalovirus (CMV) immediate-early promoter/enhancer and the nonliposomal lipid formulation FuGENE 6 (Roche Applied Sciences, Mannheim, Germany), as previously described [21]. Aliquots of chondrocytes used for the production of alginate spheres were processed for X-gal staining and demonstrated a mean transfection efficiency of $42.1 \pm 3.0 \%$, consistent with previous data using this technique [21].

\section{Encapsulation of transfected and non-transfected chondrocytes in alginate spheres}

One day after transfection, lacZ- and FGF-2-transfected chondrocytes were encapsulated in alginate as previously described [21]. Briefly, transfected chondrocytes were suspended in $1.2 \%$ alginate in $0.15 \mathrm{M} \mathrm{NaCl}$ at a density of $4 \times 10^{6}$ cells $/ \mathrm{ml}$. The cell suspension was then extruded through a 21-gauge needle (Braun, Melsungen, Germany) into $102 \mathrm{mM} \mathrm{CaCl}_{2}$ at room temperature under constant shaking and allowed to polymerize for $10 \mathrm{~min}$. The resulting alginate spheres containing lacZ- or FGF-2transfected chondrocytes (termed lacZ or FGF-2 spheres) 
were washed twice in $0.15 \mathrm{M} \mathrm{NaCl}$, followed by two washes in basal medium, and then placed in 96-well plates (one sphere/well) containing basal medium that was changed three times per week and kept at $37^{\circ} \mathrm{C}$ in a humidified atmosphere of $10 \% \mathrm{CO}_{2}$. At days $0,1,3,7,14$, 21 and 28 post-encapsulation, individual spheres were dissolved in $100 \mu \mathrm{l} 55 \mathrm{mM}$ sodium citrate, $90 \mathrm{mM} \mathrm{NaCl}$, $\mathrm{pH}$ 6.8, for $20 \mathrm{~min}$ at room temperature and submitted to further analysis. Spheres composed of non-transfected chondrocytes were individually cultured in basal medium without or with the addition of $5 \mathrm{ng} / \mathrm{ml}$ recombinant FGF2 (PeproTech, London, UK) or in growth medium. For these experiments, the complete medium was replaced every other day.

\section{Cell counts and glycosaminoglycan content of alginate spheres}

Released chondrocytes were counted and their viability was determined using a Neubauer chamber and trypan blue exclusion staining based on four counts per sample. For glycosaminoglycan analysis, released chondrocytes were incubated overnight in $125 \mu \mathrm{g} / \mathrm{ml}$ papain in PBE containing $10 \mathrm{mM} \mathrm{Na} \mathrm{N}_{2}$ ETDA. Glycosaminoglycans were measured spectrophotometrically by binding to the dimethylmethylene blue dye, using chondroitin-6sulfate as a standard [28]. Absorbance was monitored at $530 \mathrm{~nm}$. Measurements were performed using a GENios spectrophotometer/fluorometer (Tecan, Crailsheim, Germany).

\section{Transplantation of alginate spheres to osteochondral cartilage defects in vivo}

The transplantation of spheres in vivo was performed on the day of encapsulation (1 day post-transfection). All animal procedures were approved by the Saarland Governmental Animal Care Committee. Chinchilla bastard rabbits (Charles River) were kept in air-conditioned rooms with constant temperatures and a regular light/dark scheme. They received water ad libitum and were fed a standard diet. Fourteen female rabbits (mean weight: $2.9 \pm 0.3 \mathrm{~kg}$; seven animals per group) were anesthetized by intramuscular injection of Rompun
(0.2 ml/kg body weight; Bayer, Leverkusen, Germany) and Ketavet $(0.75 \mathrm{mg} / \mathrm{kg}$ body weight; Pharmacia \& Upjohn, Erlangen, Germany). The knee joint was entered using a medial parapatellar approach. The patella was dislocated laterally and the knee flexed to $90^{\circ}$. A cylindrical osteochondral defect was created in the patellar groove of each knee $(n=28$ defects) with a manual cannulated burr (3.2 mm in diameter; Synthes, Umkirch, Germany). The defects were washed with PBS to remove debris and blotted dry. Spheres (from a single preparation, Table 1) were press-fit into the defects. The right and left knees alternately received lacZ or FGF-2 spheres. The patella was reduced and the knee was put through a range of motion to assure the stability of the spheres. Incisions were closed in layers. Immediately postoperatively, animals were allowed full weight bearing without any immobilization. Animals were allowed to climb and jump 6 weeks after the implantation.

\section{Detection of transgene expression}

Detection of $\beta$-galactosidase activity was performed by X-gal staining using a standard protocol [21]. To measure the production of FGF-2 by transfected chondrocytes encapsulated in alginate, the spheres were individually cultivated in 96-well plates (one sphere/well) in growth medium. At the indicated time points, spheres were washed twice in basal medium and the medium was replaced with $200 \mu \mathrm{l}$ basal medium. After $24 \mathrm{~h}$, the conditioned medium was collected. To monitor the FGF-2 content in the synovial fluid, knees received a lavage with $1 \mathrm{ml}$ PBS. All samples were centrifuged to remove cell debris and stored at $-80^{\circ} \mathrm{C}$. Samples were analyzed using a FGF-2 enzymelinked immunosorbent assay (ELISA) (R\&D Systems, Wiesbaden, Germany) with a detection limit of 3 $\mathrm{pg} / \mathrm{ml}$.

\section{Histological and immunohistochemical analysis}

Spheres cultured in vitro were fixed in 4\% phosphatebuffered formalin, embedded in paraffin, sectioned

Table 1. FGF-2 production by chondrocyte-alginate spheres in vitro

\begin{tabular}{|c|c|c|c|c|c|c|c|}
\hline $\begin{array}{l}\text { Day post-transfection } \\
\text { Day post-encapsulation }\end{array}$ & $\begin{array}{l}1 \\
0\end{array}$ & $\begin{array}{l}2 \\
1\end{array}$ & $\begin{array}{l}4 \\
3\end{array}$ & $\begin{array}{l}8 \\
7\end{array}$ & $\begin{array}{l}15 \\
14\end{array}$ & $\begin{array}{l}22 \\
21\end{array}$ & $\begin{array}{l}29 \\
28\end{array}$ \\
\hline \multicolumn{8}{|l|}{ lacZ spheres } \\
\hline FGF-2 (ng/107 viable cells/24h) & $0.5 \pm 0.2$ & $0.5 \pm 0.1$ & $0.1 \pm 0.1$ & $0.4 \pm 0.0$ & $0.7 \pm 0.6$ & $0.5 \pm 0.4$ & $0.2 \pm 0.1$ \\
\hline $\begin{array}{l}\text { FGF-2 (pg/sphere/24 h) } \\
\text { FGF-2 spheres }\end{array}$ & $2.4 \pm 0.8$ & $2.7 \pm 0.2$ & $0.2 \pm 0.2$ & $1.9 \pm 0.1$ & $3.4 \pm 2.8$ & $2.6 \pm 2.0$ & $0.7 \pm 0.4$ \\
\hline FGF-2 (ng/10 viable cells/24 h) & $41.1 \pm 0.5$ & $47.4 \pm 1.0$ & $17.7 \pm 2.1$ & $10.7 \pm 3.5$ & $1.3 \pm 0.3$ & $4.2 \pm 2.9$ & $2.0 \pm 0.2$ \\
\hline FGF-2 (pg/sphere/24 h) & $210.4 \pm 1.7$ & $210.7 \pm 0.4$ & $77.0 \pm 14.4$ & $48.5 \pm 14.8$ & $7.6 \pm 2.0$ & $27.0 \pm 21.0$ & $11.4 \pm 0.5$ \\
\hline
\end{tabular}

Lapine articular chondrocytes transfected with pCMVlacZ or pCMVhFGF-2 were encapsulated in alginate on day 1 post-transfection and kept in basal medium for 28 days. Conditioned medium was collected at the denoted times and FGF-2 protein concentrations were measured by ELISA. Data are expressed as mean \pm standard deviation (S.D.) ( $n=2$ for each time point and condition). 
and submitted to histological and immunohistochemical analysis as described below. Three $(\mathrm{n}=7)$ and 14 weeks $(\mathrm{n}=7)$ after implantation, rabbits were euthanized with pentobarbital $(150 \mathrm{mg} / \mathrm{kg}$ body weight; Merial, Hallbergmoos, Germany) and the knee joints were exposed and examined for synovitis, osteophytes, or other reactions. The appearance of the defect (color, integrity, contour) and the articulating surfaces were documented. Distal femurs were retrieved, fixed in 4\% phosphatebuffered formalin, trimmed, and decalcified. Paraffinembedded frontal sections $(5 \mu \mathrm{m})$ were stained with safranin-O and hematoxylin and eosin (H\&E) according to routine histological protocols [29]. For FGF-2, type II and type I collagen immunostaining, deparaffinized sections were submerged for $30 \mathrm{~min}$ in $0.3 \%$ hydrogen peroxide. After washing with PBS and incubation in $0.1 \%$ trypsin for $30 \mathrm{~min}$, sections were washed with PBS and blocked with $3 \%$ bovine serum albumin in PBS (blocking buffer) for $30 \mathrm{~min}$. The sections then were incubated with a 1:100 dilution of a monoclonal mouse antiFGF-2 IgG (Santa Cruz Biotechnology, Santa Cruz, CA, USA) or with a $1: 50$ dilution of a monoclonal mouse anti-type-II or anti-type-I collagen IgG (Acris Antibodies, Hiddenhausen, Germany) in blocking buffer for $24 \mathrm{~h}$ at $4^{\circ} \mathrm{C}$, washed and exposed to a $1: 500$ dilution of a biotinylated anti-mouse antibody (Vector Laboratories, Grünberg, Germany) for $1 \mathrm{~h}$ at room temperature. After washing with PBS, the sections were incubated for $30 \mathrm{~min}$ with the avidin-biotin-peroxidase reagent (Vectastain Elite ABC kit; Vector Laboratories), washed, and exposed to diaminobenzidine (Vector Laboratories). To control for secondary immunoglobulins, sections were processed as above, except for the secondary antibody.

\section{Evaluation of histological sections}

The synovial membrane was evaluated using a previously published scoring system [30,31]. The categories of the score include villus thickening (fibrosis), villus architecture (blunting), and the presence of inflammatory cell infiltrates. The area occupied by lacZ and FGF-2 spheres in the defects was measured on H\&E-stained serial histological sections of the distal femora that were taken within approximately $0.6 \mathrm{~mm}$ from the center of the defects at $200 \mu \mathrm{m}$ intervals $(\mathrm{n}=3-5$ per defect). Low-magnification images of the cartilage defects were acquired by a solid-state CCD camera mounted on a BX-45 microscope (Olympus, Hamburg, Germany). The image on the monitor was digitalized and the border of the sphere of each section was traced by a blinded observer. The area of the sphere was calculated with the analySIS program (Soft Imaging System, Münster, Germany). Immunoreactivity to type II collagen in the repair tissue was compared with that of the adjacent normal articular cartilage which served as a positive internal control. Immunoreactivity to type I collagen in the repair tissue was compared to that of the subchondral bone adjacent to the normal articular cartilage which served as a positive internal control. A score was given to each knee: -, no immunoreactivity; +, weaker immunoreactivity; ++, similar immunoreactivity; +++ , stronger immunoreactivity. For the quantitative assessment of the repair tissue, serial histological sections of the distal femora were taken at $200 \mu \mathrm{m}$ intervals. Sections within approximately $1.0 \mathrm{~mm}$ from the center of the defect ( $n=9-10$ per defect) were analyzed using the articular cartilage repair scoring system described by Sellers and co-workers [32,33]. Specific parameters that were evaluated include filling of the defect, integration of the new cartilage, safranin O staining, cell morphology, architecture within the defect and of its surface, restoration of the subchondral bone and tidemark. Scores were combined and resulted in an average total score. Values range from 31 points (empty defect without repair tissue) to 0 points (normal articular cartilage, complete regeneration). A total of 274 sections were independently scored by three individuals without knowledge of the treatment groups.

\section{Statistical analysis}

Each test condition was performed in duplicate for the in vitro characterization experiments and with seven defects per group and time point for the in vivo experiments. Differences in cell number, viability and glycosaminoglycan content were compared between treatment and control groups using nested analysis of variance (ANOVA). To determine the sample size for the in vivo experiments, a standard deviation of $25 \%$ for the mean total score was estimated based on literature values for selected cartilage repair procedures. For a power of $80 \%$ (beta $=0.20$ ) and a two-tailed alpha level of 0.05 , a sample size of six animals per group would have been sufficient to detect a mean difference of 5 points between the groups, assuming a pooled standard deviation of 2.5 points [effect size $=5 / 2.5=2.0$, using the two-sample Student's $t$ test (version 5.0, nQuery Advisor, Statistical Solutions, Saugus, MA, USA)]. To evaluate the in vivo experiments, points for each category and total score were compared between the two groups using a mixed general linear model with repeated-measures (knees nested within the same animals). All continuous variables, including FGF2 production and histological scoring for parameters such as cell morphology, architecture and tidemark, were tested for normality using the Kolmogorov-Smirnov goodness-of-fit method and no significant skewness or kurtosis was detected. Therefore, continuous data are expressed in terms of the mean \pm standard deviation (S.D.) or mean $\pm 95 \%$ confidence interval. A twotailed $P<0.05$ was considered statistically significant. Statistical analysis of the data was performed using the SPSS software package (version 12.0, SPSS Inc., Chicago, IL, USA). 


\section{Results}

\section{Human FGF-2 is efficiently released from alginate spheres over a prolonged period of time in vitro}

The ability of the alginate spheres to release the recombinant factor produced by the encapsulated cells is a prerequisite for the localized delivery of the gene product to cartilage defects in vivo. Accordingly, we transfected lapine articular chondrocytes with expression plasmid vectors carrying either the $E$. coli lacZ gene or a human FGF-2 cDNA, using the nonliposomal lipid preparation FuGENE 6. One day after transfection, the modified chondrocytes were encapsulated in alginate. The resulting lacZ and FGF-2 spheres were kept in culture for 28 days and the course of FGF-2 secretion was assessed over time. In vitro, maximal FGF-2 production of FGF-2 spheres was $47.4 \pm 1.0 \mathrm{ng} / 10^{7}$ viable chondrocytes $/ 24 \mathrm{~h}$ at day 2 after transfection, 95-fold higher compared to lacZ spheres $(P<0.001, \mathrm{n}=2)$ (Table 1$)$. After 28 days in vitro, FGF-2 production of FGF-2 spheres was $2.0 \pm 0.2 \mathrm{ng} / 10^{7}$ viable chondrocytes $/ 24 \mathrm{~h}, 10$-fold higher than in lacZ spheres $(P=0.006, \mathrm{n}=2)$.

\section{Overexpression of human FGF-2 stimulates chondrocyte proliferation but not glycosaminoglycan synthesis in alginate spheres in vitro}

Next, the biological effects of human FGF-2 overexpression on the encapsulated chondrocytes were investigated. At different time points in vitro, viability, cell number and glycosaminoglycan content in the spheres were determined. At the time of encapsulation, the chondrocytes in the $l a c Z$ and FGF-2 spheres had a viability of $89.0 \pm 1.4 \%$ and $91.5 \pm 0.7 \%$, respectively $(P>0.05, \mathrm{n}=2)$. Cell viability remained above $80 \%$ in all spheres at all other time points (data not shown). At day 29 post-transfection, the viability of the chondrocytes in the lac $Z$ and FGF2 spheres was similar compared to day 1 ( $84.9 \pm 2.4 \%$ and $89.0 \pm 1.7 \%$, respectively; $P>0.05, \mathrm{n}=10$ ). The number of viable chondrocytes in the lacZ and FGF-2 spheres was similar at the time of encapsulation, 1 day post-transfection $\left(4.9 \pm 0.2 \times 10^{4}\right.$ and $5.1 \pm 0.1 \times 10^{4}$ cells/sphere, respectively; $P>0.05, \mathrm{n}=2$ ). At day 29 post-transfection, the number of viable chondrocytes in lacZ spheres increased by $25.5 \%$ to $6.2 \pm 0.8 \times 10^{4}$ cells/sphere, without reaching statistical significance when compared to day $1(P>0.05, \mathrm{n}=10)$. In contrast, FGF-2 spheres contained $7.1 \pm 0.4 \times 10^{4}$ cells/sphere, significantly more when compared to lac $Z$ spheres at this time point $(P<0.001, \mathrm{n}=10)$. This corresponds to a $39.2 \%$ increase $(P=0.008, \mathrm{n}=10)$ in the number of viable chondrocytes in FGF-2 spheres between days 1 and 29 post-transfection. At day 29, the glycosaminoglycan content of FGF-2 spheres was not different from that of lacZ spheres $(8.17 \pm 0.84$ and $8.46 \pm 1.09 \mu \mathrm{g} /$ sphere, respectively; $P>0.05, \mathrm{n}=8$ ).

\section{Exogenous FGF-2 stimulates the proliferation of chondrocytes in alginate spheres in vitro}

In order to compare the increase in cell proliferation as a result of FGF-2 overexpression to that obtained after exposure to exogenous FGF-2 protein, non-transfected chondrocytes encapsulated in alginate were incubated in basal medium without or with $5 \mathrm{ng} / \mathrm{ml} \mathrm{FGF-2} \mathrm{or} \mathrm{in}$ growth medium (as a positive control) for 28 days. At the time of encapsulation, chondrocytes had a viability of $88.0 \pm 4.5 \%$ and a total viable cell number of $5.9 \pm 0.6 \times$ $10^{4}$ cells/sphere. After the 28-day incubation in basal medium, cell viability was $84.1 \pm 2.4 \%$ and the number of viable chondrocytes was $6.1 \pm 0.4 \times 10^{4}$ cells/sphere. This corresponds to a $3.4 \%$ increase in the number of viable cells, not significantly different compared to day 0 $(P>0.05$ for viability and number of viable chondrocytes in comparison to day $0, \mathrm{n}=10$ ). Continuous exposure to $5 \mathrm{ng} / \mathrm{ml}$ exogenous FGF-2 led to a cell viability of $89.0 \pm$ $1.3 \%$ and to $7.9 \pm 1.1 \times 10^{4}$ viable chondrocytes/sphere after 28 days. This corresponds to a $33.9 \%$ increase in the number of viable chondrocytes compared to day $0(P<0.01, \mathrm{n}=10)$. When chondrocyte-alginate spheres were continuously exposed to growth medium, chondrocyte viability was $90.0 \pm 1.3 \%$ and the number of viable chondrocytes was $8.5 \pm 0.7 \times 10^{4}$ cells/sphere at day 28. This corresponds to a $44.1 \%$ increase in the number of viable chondrocytes $(P=0.01, \mathrm{n}=10)$. A comparison of the different culture conditions at day 28 after encapsulation revealed no significant differences in cell viability and in the number of viable chondrocytes between spheres incubated in basal medium containing $5 \mathrm{ng} / \mathrm{ml} \mathrm{FGF-2}$ or in growth medium $(P<0.05, \mathrm{n}=10$ spheres). Compared with spheres incubated in basal medium alone, chondrocyte viability and the number of viable cells were significantly higher $(P<0.01, \mathrm{n}=10$ spheres) in spheres incubated in $5 \mathrm{ng} / \mathrm{ml} \mathrm{FGF-2} \mathrm{or} \mathrm{in}$ growth medium. Thus, the $39.2 \%$ increase following FGF2 overexpression is well comparable to the $33.9 \%$ increase following continuous exposure to $5 \mathrm{ng} / \mathrm{ml}$ exogenous FGF-2 protein in vitro.

\section{Localized human FGF-2 gene transfer does not induce a synovial inflammatory response in vivo}

On the day of encapsulation, lacZ or FGF-2 spheres were press-fit into osteochondral defects in each patellar groove of 14 rabbits. Three and 14 weeks following implantation, there were no macroscopic signs of inflammation or hematoma. FGF-2 concentrations were monitored in the lavage fluid of knees. After 3 weeks, there was no difference between knees receiving lacZ or FGF-2 spheres 
Table 2. Effects of FGF-2 gene transfer at 3 and 14 weeks in vivo on histological grading of the synovium

\begin{tabular}{lccc}
\hline Category & lacZ spheres & FGF-2 spheres & $P$ value \\
\hline 3 weeks & & & \\
Villus thickening & $0.43 \pm 0.50$ & $0.46 \pm 0.51$ & $>0.05$ \\
Villus architecture & $0.29 \pm 0.46$ & $0.25 \pm 0.44$ & $>0.05$ \\
Inflammatory cell infiltrate & $0.11 \pm 0.31$ & $0.14 \pm 0.36$ & $>0.05$ \\
\hline Average total score & $0.82 \pm 0.16$ & $0.86 \pm 0.16$ & $>0.05$ \\
\hline 14 weeks & & & \\
Villus thickening & $0.14 \pm 0.36$ & $0.14 \pm 0.36$ & $>0.05$ \\
Villus architecture & $0.29 \pm 0.49$ & $0.21 \pm 0.43$ & $>0.05$ \\
Inflammatory cell infiltrate & $0.00 \pm 0.00$ & $0.14 \pm 0.36$ & $>0.05$ \\
\hline Average total score & $0.43 \pm 0.14$ & $0.50 \pm 0.04$ & $>0.05$ \\
\hline
\end{tabular}

Each category or total score is calculated as the average score performed by two independent evaluators. Points for each category and total score were compared between the FGF-2 and lacZ groups using ANOVA. Data are expressed as mean \pm S.D.

$(24.3 \pm 23.6$ and $21.5 \pm 13.0 \mathrm{pg} / \mathrm{ml}$, respectively; $P>$ $0.05, \mathrm{n}=7)$. Similar results were obtained after 14 weeks in vivo $(66.7 \pm 55.6$ and $58.0 \pm 25.7 \mathrm{pg} / \mathrm{ml}$, respectively; $P>0.05, \mathrm{n}=7)$. We next investigated the effects of the recombinant human FGF-2 overexpression on the synovial membrane using a previously published score. Analysis of the thickness, architecture of synovial villi, and presence of inflammatory cell infiltrates revealed no significant differences between knees receiving lacZ or FGF-2 spheres at either 3 or 14 weeks $(P>0.05$ for both time points, $\mathrm{n}=7$ ) (Table 2; Figures 2 and $3 \mathrm{~L}-3 \mathrm{M}$ ).

\section{Alginate spheres containing genetically modified chondrocytes produce FGF-2 for at least 3 weeks in vivo and are progressively resorbed}

Over the course of the in vivo experiment, the spheres remained underneath the original articular cartilage (Figures 2 and 3A, 3B). After 3 weeks, both lacZ and FGF-2 spheres were surrounded and in part invaded by a resorptive tissue consisting of undifferentiated mesenchymal cells, histiocytotic cells and lymphocytes (Figure 1A and 1B). FGF-2 expression was detected by immunohistochemistry in FGF-2 spheres (Figure 1F). The areas occupied by lacZ and FGF-2 spheres after 3 weeks were $1.62 \pm 1.23$ and $1.14 \pm 0.63 \mathrm{~mm}^{2}$, respectively $(P>$ $0.05, \mathrm{n}=7$ ). After 14 weeks, remnants of the spheres were detectable in the newly formed subchondral bone (Figures 1C and 1D). Spheres were surrounded by a loose reparative tissue that consisted of fibroblasts and histiocytes, consistent with remnants of a resorptive granulation tissue. The areas occupied by lac $Z$ and FGF-2 spheres were $0.03 \pm 0.05$ and $0.08 \pm 0.13 \mathrm{~mm}^{2}$, respectively $(P>0.05, \mathrm{n}=3$ ). Between 3 and 14 weeks, the spheres were progressively resorbed, as indicated by a significant decrease in their area $(P<0.0001$ for both groups).
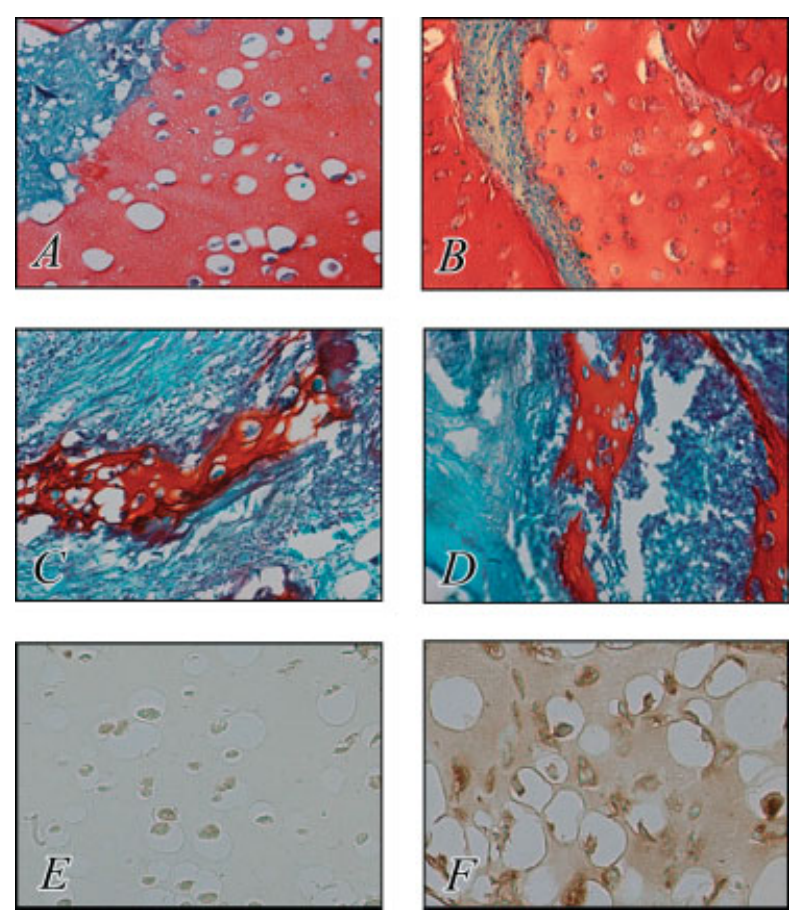

Figure 1. Representative histological sections of lacZ (A, C, E) and FGF-2 spheres (B, D, F) after 3 (A, B, E, F) and 14 weeks $(C, D)$ in vivo stained with safranin $O(A-D)$ and a monoclonal mouse anti-human FGF-2 IgG. At 3 weeks, spheres were surrounded and in part invaded by a resorptive tissue (A, B). FGF-2 was expressed in FGF-2 spheres (F). After 14 weeks, only remnants of the spheres were detectable, which were surrounded by a resorptive granulation tissue $(C, D)$. Original magnification $\times 200(\mathrm{~A}-\mathrm{D})$ and $\times 250(\mathrm{E}, \mathrm{F})$

\section{Effect of localized human FGF-2 gene transfer on type II and type I collagen synthesis at 3 and 14 weeks in vivo}

The new tissue in the cartilage defects was analyzed by immunohistochemistry for the presence of type II collagen, a major component of the extracellular matrix of hyaline articular cartilage. Three weeks after transplantation, immunoreactivity to type II collagen was weaker in the repair tissue of defects that received lacZ spheres than in those where FGF-2 spheres were applied (Table 3; Figures $2 \mathrm{G}$ and $2 \mathrm{H}$ ). Fourteen weeks after transfection, immunoreactivity to type II collagen in defects receiving lac $Z$ spheres was less than or similar to that of the adjacent normal articular cartilage (Table 3; Figures $3 \mathrm{G}$ and $3 \mathrm{H}$ ). All defects receiving FGF-2 spheres exhibited immunoreactivity to type II collagen similar to that of the adjacent normal articular cartilage. The new tissue was then analyzed by immunohistochemistry for the presence of type I collagen, an indicator of fibrocartilage. Three weeks after transplantation, immunoreactivity to type I collagen was stronger in the repair tissue of defects that received lac $Z$ spheres than in those where FGF-2 spheres were applied (Table 4; Figures 2I- 2K). Fourteen weeks after transfection, immunoreactivity to type I collagen decreased in defects of both groups, while 

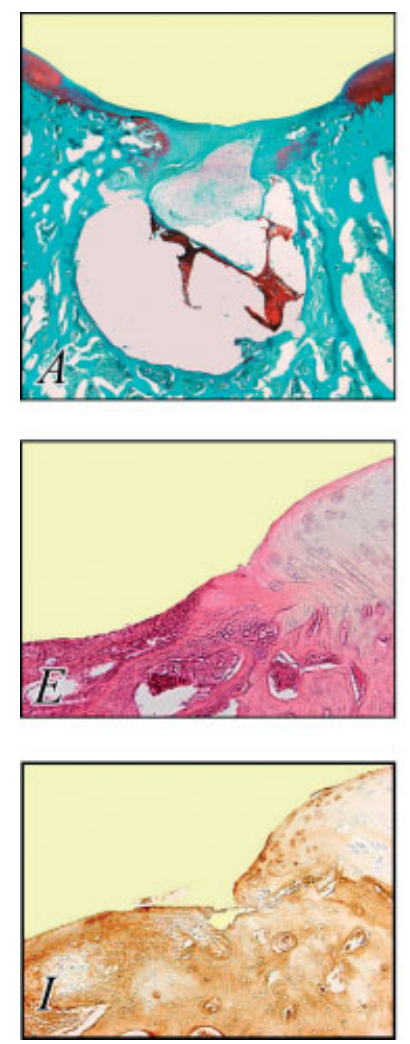
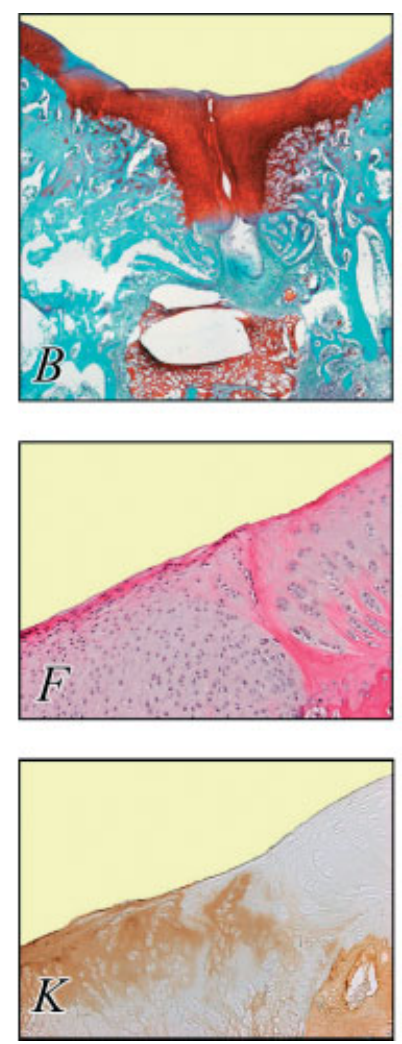
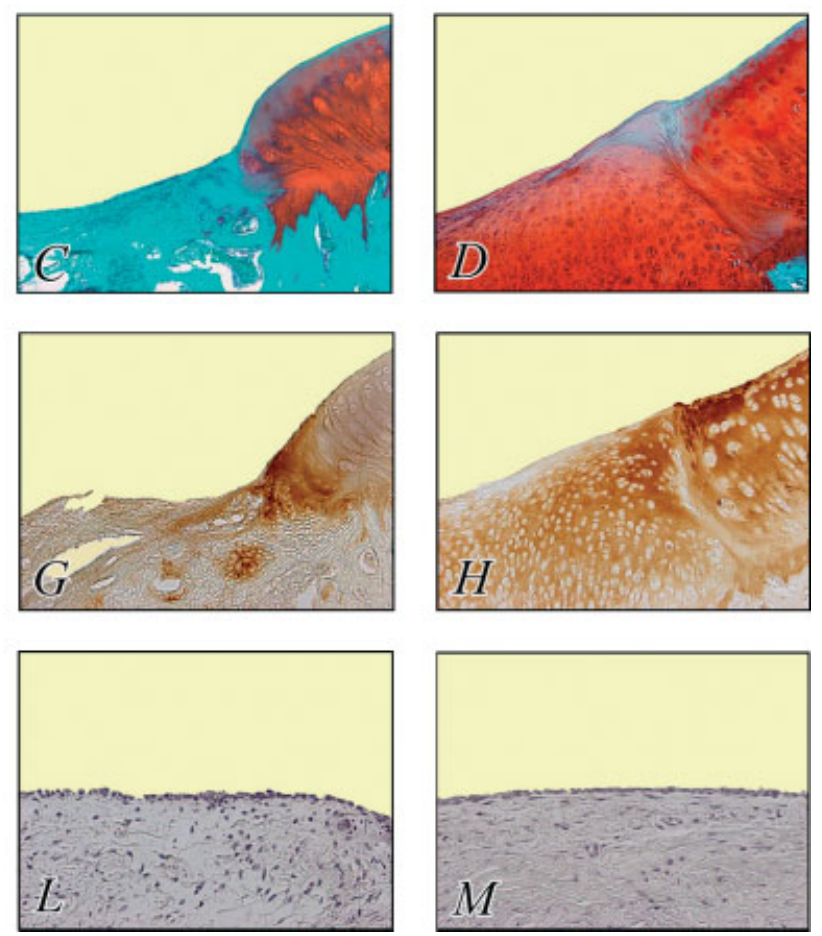

Figure 2. Stimulation of chondrogenesis in articular cartilage defects 3 weeks after transplantation of genetically modified chondrocytes in vivo. Histological appearance of osteochondral defects following treatment with a lacZ (A, C, E, G, I, L) or a FGF-2 sphere (B, D, F, H, K, M) stained with safranin O (A-D), hematoxylin and eosin (H\&E) (E, F), a monoclonal mouse anti-human type-II collagen IgG (G, H), or a monoclonal mouse anti-human type I collagen IgG (I, K). Images (L) and (M) depict the synovial membrane adjacent to the cartilage (H\&E). Images (C) and (D) are magnified views of images (A) and (B) illustrating the area of integration between the repair tissue (left-hand side of each picture) with the adjacent normal articular cartilage (right-hand side of each picture). Spheres remained in a subchondral location and are visible at the bottom of images (A) and (B). Safranin $O$ staining was increased in defects receiving a FGF-2 sphere (B, D). Cells in the repair tissue of the osteochondral defect following treatment with a lacZ sphere were spindle-shaped, indicative of a fibrous repair tissue (C, E). In contrast, cells in the new tissue in the defect following treatment with a FGF-2 sphere were round and had round nuclei, thus exhibiting characteristics of chondrocytes (D, F). Immunoreactivity to type II collagen was already present in the repair tissue of the defect receiving a FGF-2 sphere (H). Sections were taken from defects having a histological rating equal to the mean score for its respective treatment group. Photomicrographs were obtained using standardized photographic parameters, including light intensity. Original magnifications $\times 40(A, B)$ or $\times 100$ (C-M)

Table 3. Semiquantitative analysis of type II collagen immunoreactivity in the repair tissue 3 and 14 weeks in vivo

\begin{tabular}{lccllll}
\hline \multirow{2}{*}{$\begin{array}{l}\text { Animal } \\
\text { number }\end{array}$} & \multicolumn{2}{c}{3 weeks } & & \multicolumn{2}{c}{14 weeks } \\
\cline { 2 - 3 } \cline { 5 - 6 } \cline { 5 - 6 } & lacZ spheres & FGF-2 spheres & & lacZ spheres & FGF-2 spheres \\
\hline 1 & + & ++ & & + & ++ \\
2 & ++ & ++ & & ++ & ++ \\
3 & + & ++ & & ++ & ++ \\
4 & ++ & + & & ++ & ++ \\
5 & - & ++ & & ++ & ++ \\
6 & - & +++ & & + & ++ \\
7 & ++ & + & & ++ & ++ \\
\hline Range & - to ++ & + to +++ & & + to ++ & ++ \\
\hline
\end{tabular}

Type II collagen immunoreactivity in the repair tissue of the defect was compared to that of the normal articular cartilage adjacent to the defect, used as a positive internal control. Type II collagen immunoreactivity was scored as follows: - , no immunoreactivity; + , weaker immunoreactivity; ++ , similar immunoreactivity; +++ , stronger immunoreactivity.

it continued to be stronger in defects that received lacZ spheres (Table 4; Figures 3I-3K).

\section{Localized human FGF-2 gene transfer stimulates chondrogenesis at 3 and 14 weeks in vivo}

To study the effects of FGF-2 overexpression on articular cartilage repair in vivo, the newly formed repair tissue within the defect was evaluated using a previously published grading system [32,33] that consists of eight individual parameters. First, the animal model was validated to confirm the absence of a complete cartilage regeneration. The development over time of the new tissue was determined for defects treated with lacZ spheres. A comparison for defect healing of the lacZ control group between 3 and 14 weeks in vivo revealed a significantly better healing at 14 weeks with respect to filling of the defect $(P<0.01)$, architecture within the defect $(P<0.05)$, and average total score $(P<0.01)$, indicating a maturation of the repair tissue over time. In parallel, signs of degradation of the repair tissue were evident after 14 weeks in vivo, indicated by a higher 

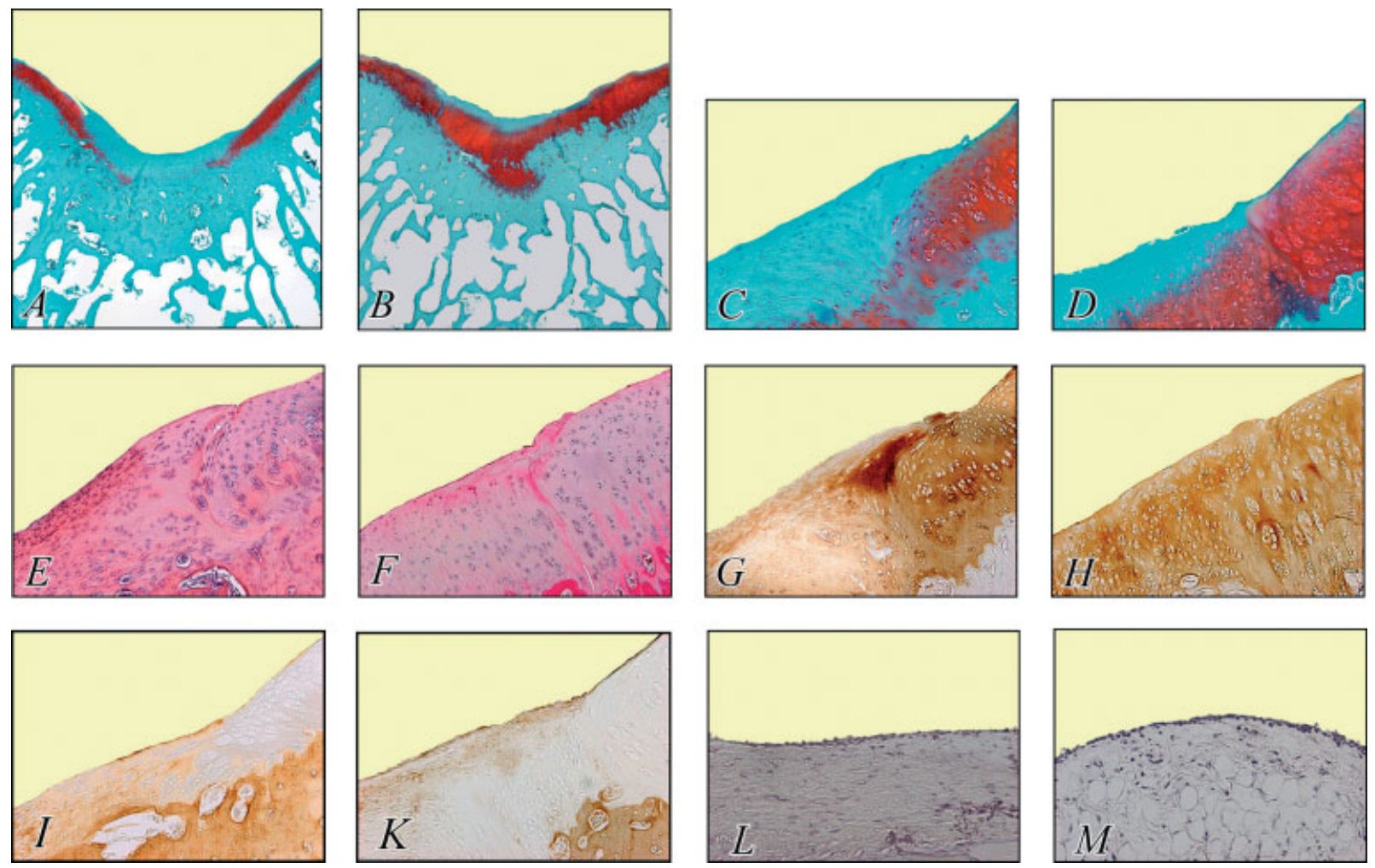

Figure 3. Stimulation of chondrogenesis in articular cartilage defects 14 weeks after transplantation of genetically modified chondrocytes in vivo. Histological appearance of osteochondral defects following treatment with a lacZ (A, C, E, G, I, L) or a FGF-2 sphere (B, D, F, H, K, M) stained with safranin O (A-D), hematoxylin and eosin (H\&E) (E, F), a monoclonal mouse anti-human type II collagen IgG (G, H), or a monoclonal mouse anti-human type I collagen IgG (I, K). Images (L) and (M) depict the synovial membrane adjacent to the cartilage (H\&E). Images (C) and (D) are magnified views of images (A) and (B). Safranin $O$ staining is increased in the defect receiving a FGF-2 sphere (B, D) compared to the defect receiving a lacZ sphere. Cells in the repair tissue of both defects were round and had round nuclei (C-F). However, only cells in the defect treated with a FGF-2 sphere were oriented in a columnar fashion (F). Immunoreactivity to type II collagen was reduced in the repair tissue of the defect receiving a lacZ sphere (G). Sections were taken from defects having a histological rating equal to the mean score for its respective treatment group. Photomicrographs were obtained using standardized photographic parameters, including light intensity. Original magnifications $\times 40(\mathrm{~A}, \mathrm{~B})$ or $\times 100(\mathrm{C}-\mathrm{M})$

(lower) score value for both the surface of the new tissue and the tidemark compared to 3 weeks (Tables 5 and 6).

Subsequently, articular cartilage repair was compared for defects receiving lacZ or FGF-2 spheres 3 and 14 weeks after implantation. No complete articular cartilage

Table 4. Semiquantitative analysis of type I collagen immunoreactivity in the repair tissue 3 and 14 weeks in vivo

\begin{tabular}{|c|c|c|c|c|}
\hline \multirow{2}{*}{$\begin{array}{l}\text { Animal } \\
\text { number }\end{array}$} & \multicolumn{2}{|c|}{3 weeks } & \multicolumn{2}{|c|}{14 weeks } \\
\hline & lacZ spheres & FGF-2 spheres & lacZ spheres & FGF-2 spheres \\
\hline 1 & - & + & + & + \\
\hline 2 & + & + & - & - \\
\hline 3 & + & - & ++ & - \\
\hline 4 & + & ++ & - & - \\
\hline 5 & - & - & - & - \\
\hline 6 & ++ & - & + & - \\
\hline 7 & ++ & - & - & - \\
\hline Range & - to ++ & - to ++ & - to ++ & - to + \\
\hline
\end{tabular}

Type I collagen immunoreactivity in the repair tissue of the defect was compared to that of the subchondral bone adjacent to the normal articular cartilage, used as a positive internal control. Type I collagen immunoreactivity was scored as follows: - , no immunoreactivity; + , weaker immunoreactivity; ++ , similar immunoreactivity; +++ , stronger immunoreactivity. regeneration ( 0 points) was achieved at both time points for defects treated with lacZ and FGF-2 spheres. However, after 3 weeks, the defects receiving FGF-2 spheres were

Table 5. Effects of FGF-2 gene transfer at 3 weeks on histological grading of the repair tissue

\begin{tabular}{lccc}
\hline Category & $\begin{array}{c}\text { lacZ spheres } \\
\text { Mean }(95 \% \mathrm{Cl})\end{array}$ & $\begin{array}{c}\text { FGF-2 spheres } \\
\text { Mean }(95 \% \mathrm{Cl})\end{array}$ & $P$ value \\
\hline $\begin{array}{l}\text { Filling of the defect } \\
\text { Integration }\end{array}$ & $2.00(0.83-3.17)$ & $0.77(0.27-1.27)$ & $<0.01^{* *}$ \\
$\begin{array}{l}\text { Matrix staining } \\
\text { Cell morphology }\end{array}$ & $2.50(0.81-2.19)$ & $1.41(1.02-1.81)$ & 0.25 \\
$\begin{array}{l}\text { Architecture within the } \\
\text { defect }\end{array}$ & $2.96(2.00-3.32)$ & $2.14(0.79-3.50)$ & 0.07 \\
$\begin{array}{l}\text { Architecture of the } \\
\text { surface }\end{array}$ & $1.64(0.27-3.04)$ & $1.67(1.59-2.98)$ & 0.12 \\
Subchondral bone & $2.23(0.96-2.15)$ & $<0.01^{* *}$ \\
Tidemark & $3.84(3.56-4.12)$ & $1.52(1.53(1.03-2.63)$ & 0.59 \\
\hline Average total score & $19.1(15.6-22.6)$ & $15.2(11.6-18.8)$ & $<0.01^{* *}$ \\
\hline
\end{tabular}

Each category or total score is based on the average of three independent evaluators. Points for each category and total score were compared between the FGF- 2 and lacZ groups using a mixed-model analysis with repeated-measures (knees nested within the same animals; $\mathrm{Cl}=$ confidence interval). Means indicate the estimated scores in points for each category. 
Table 6. Effects of FGF-2 gene transfer at 14 weeks on histological grading of the repair tissue

\begin{tabular}{|c|c|c|c|}
\hline Category & $\begin{array}{l}\text { lacZ spheres } \\
\text { Mean }(95 \% \mathrm{Cl})\end{array}$ & $\begin{array}{l}\text { FGF-2 spheres } \\
\text { Mean }(95 \% \mathrm{Cl})\end{array}$ & $P$ value \\
\hline Filling of the defect & $0.48(0.09-0.87)$ & $0.11(0.01-0.38)$ & 0.08 \\
\hline Integration & $1.33(0.68-1.98)$ & $1.18(0.95-1.42)$ & 0.61 \\
\hline Matrix staining & $2.52(1.63-3.41)$ & $1.52(0.72-2.33)$ & 0.07 \\
\hline Cell morphology & $2.60(1.82-3.38)$ & $1.40(1.04-1.76)$ & $<0.01^{* *}$ \\
\hline $\begin{array}{l}\text { Architecture within the } \\
\text { defect }\end{array}$ & $1.60(0.90-2.30)$ & $1.18(0.76-1.61)$ & 0.24 \\
\hline $\begin{array}{l}\text { Architecture of the } \\
\text { surface }\end{array}$ & $1.89(1.30-2.48)$ & $1.57(1.17-1.97)$ & 0.29 \\
\hline Subchondral bone & $1.13(0.63-1.63)$ & $0.84(0.21-1.46)$ & 0.39 \\
\hline Tidemark & $4.00(4.00-4.00)$ & $3.30(2.79-3.81)$ & $<0.01^{* *}$ \\
\hline Average total score & $15.8(13.3-18.2)$ & $11.1(8.9-13.2)$ & $<0.01^{* *}$ \\
\hline
\end{tabular}

Each category or total score is based on the average of three independent evaluators. Points for each category and total score were compared between the FGF-2 and lacZ groups using a mixed-model analysis with repeated-measures (knees nested within the same animals; $\mathrm{Cl}=$ confidence interval). Means indicate the estimated scores in points for each category.

filled with new tissue to a level similar to that of the surrounding normal articular cartilage (Figure 2B). This corresponds to the lowest (best) average individual score value (0.77) of all categories evaluated, 2.6-fold better than defects receiving lac $Z$ spheres $(P<0.01$, $\mathrm{n}=7$ ) (Table 5). Similarly, the individual score value for architecture of the defect was 1.3-fold improved for defects receiving FGF-2 spheres compared to those where lacZ spheres were applied $(P<0.01, \mathrm{n}=7)$ (Figures 2D-2F). At this early time point, the values for all other individual categories were improved for defects receiving FGF-2 spheres, compared to those that received lacZ spheres, although they did not reach statistical significance. Individual scores were combined and the resulting average total score was compared between the two groups using a mixed general linear model of statistical analysis. The average total score after 3 weeks was significantly improved for defects receiving FGF-2 spheres compared with defects receiving lacZ spheres (19.1 vs. $15.2, P<0.01, \mathrm{n}=7$ ).

After 14 weeks, the morphology of the cells repopulating the defects that received FGF-2 spheres was significantly improved compared to the control, as noted by a 1.9-fold better score value (Table 6). This indicates that the cells in the new tissue were mostly round and predominantly arranged in a columnar fashion, exhibiting characteristics of a chondrocyte phenotype (Figures 3D-3F). Re-establishment of the tidemark was significantly accelerated in the defects receiving FGF-2 spheres, compared to those where lacZ spheres were implanted. Values for all other individual parameters were improved for defects receiving FGF-2 spheres compared with the controls, although they did not reach statistical significance. The average total score after 14 weeks was significantly improved for the defects receiving FGF-2 spheres, compared with the defects that received lac $Z$ spheres (15.8 vs. 11.1; $P<0.01$, $\mathrm{n}=7$ ).

\section{Discussion}

A major challenge of gene therapy for human articular cartilage defects is to locally and safely deliver and express the therapeutic transgene. In the present study, we tested the hypothesis that overexpression of human FGF-2 via a nonviral gene delivery method by transplanted articular chondrocytes enhances the repair of full-thickness cartilage defects in vivo. The data indicate that FGF-2-transfected chondrocytes embedded in alginate spheres released functionally active FGF-2 for at least 1 month in vitro. The secreted factor selectively stimulated chondrocyte proliferation in spheres in vitro. The data further suggest that FGF-2 was expressed for at least 3 weeks in vivo without leading to differences in FGF2 concentrations in the synovial fluid or adverse effects on the synovial membrane. Finally, the data demonstrate that transplantation of FGF-2 spheres into cartilage defects augments specific parameters of chondrogenesis and results in an enhanced articular cartilage repair for a period of 14 weeks in vivo.

The delivery of FGF-2 to articular cartilage defects remains a problem. Studies that applied FGF-2 to articular cartilage defects by intraarticular injection of the protein have employed high single doses of $7 \times 10^{5} \mathrm{pg}$ [13] or between $3.6 \times 10^{3}$ [14] and $12 \times 10^{3} \mathrm{pg} /$ day [34] via osmotic pumps for up to 2 weeks. However, FGF-2 protein has a plasma half-life of less than $1 \mathrm{~h}$ and is rapidly cleared after a few hours of intraarticular administration [18]. The spheres employed in the present study secreted 210 pg FGF-2/sphere/day on day 2 and 11 pg FGF-2/sphere on day 28. These values are 10- to 100 -fold higher than the 1-2 pg of FGF-2/day secreted by stably transfected NIH3T3 cells encapsulated in a biocompatible polymer [35].

FGF-2 has been implicated in many studies as a modulator of chondrocyte proliferation in vitro $[7,8]$. In the present study, the number of viable cells in FGF-2 spheres increased by $39.2 \%$ after 28 days in vitro. This compares favorably with the $33.9 \%$ increase obtained when spheres composed of non-transfected chondrocytes were continuously exposed to $5 \mathrm{ng} / \mathrm{ml}$ exogenous FGF2 protein. Thus, the proliferative response of the encapsulated chondrocytes to FGF-2 overexpression is relevant. It remains to be determined if the amplitude of this response may be further increased, for example via strategies that lead to higher levels of the transcript. The absence of an effect of FGF-2 on the glycosaminoglycan content is consistent with the context dependence (e.g. monolayer vs. three-dimensional cell culture systems) of the actions of FGF-2. These data are in good agreement with previous findings [15,27] and suggest that the recombinant human FGF-2 protein is bioactive and capable of stimulating chondrocyte mitotic activity.

Alginate spheres can be manufactured to carry a variety of therapeutic cells [38,39] and have been used in human studies [40]. We employed alginate as a carrier for chondrocytes because it allows the safe delivery 
of genetically modified chondrocytes into osteochondral defects in vivo [21,25] and promotes the expression of cartilage-specific genes $[41,42]$. In the present study, the alginate spheres were progressively resorbed over time. This removal of the transplanted spheres may be due to a host reaction against the alginate, the transplanted allogeneic chondrocytes or the transgene. These findings are in agreement with other studies $[25,43]$ and suggest that the main role of the transplanted chondrocytes is the production of the therapeutic FGF-2, rather than to participate in the repopulation of the defect.

Local application of chondrocytes secreting human FGF-2 via spheres to cartilaginous defects may avoid a potential dilution of the factor in the synovial fluid and/or its uptake by synovial cells. It may thus prevent the development of an inflammation of the knee joint. Indeed, by 3 weeks in vivo there was a sustained production of FGF-2 by spheres that may have lasted for more than 4 weeks as previously determined using a reporter gene [21]. The absence of elevated FGF-2 levels in the synovial fluid at 3 weeks is probably due to the containment of the transgene product within the spheres and the new tissue in the defect. This supports the hypothesis of local growth factor production and action and suggests that this method of ex vivo gene delivery does not elicit any undesirable immune response. The decline of FGF-2 transgene expression in vitro has been recently demonstrated using a recombinant adeno-associated viral (rAAV) vector in a similar system [44]. FGF-2 is a secreted factor that remains mostly attached to its receptor at the surface of the genetically modified cells. The levels of FGF-2 might thus be underestimated by the ELISA. A lack of elevated intraarticular FGF-2 and of inflammatory changes of the synovial membrane may be advantageous in a clinical setting, in order to avoid undesired effects of the therapeutic factor such as observed in synovial hyperplasia [36,37].

The influence of FGF-2 on the control of cartilage growth and differentiation during embryogenesis is well established [9,10]. More recently, therapeutic effects of FGF-2 protein application on repair of full-thickness defects have been described [12-15,18]. In a fullthickness defect model, ex vivo transduced cells containing a rAAV FGF-2 vector improved cartilage repair when applied in a type I collagen gel under a periosteal flap [45]. Alternatively, direct gene delivery of FGF-2 via rAAV into cartilage defects [26] may prove even more beneficial [44]. In the present study, differences in articular cartilage repair were seen as early as 3 weeks post-transplantation. The improvement of the average total score between 3 and 14 weeks suggests that the effects of FGF-2 remain present for a period of up to 14 weeks. Although the rabbits were in their late juvenile stage at the time of implantation and adult at the time of sacrifice, the intrinsic healing of the defects was insufficient in this model system, as evidenced by the high (lower) score values in the lacZ control group and the presence of degenerative changes after 14 weeks post-operation. While defects with a diameter larger than the $3.2 \mathrm{~mm}$ employed in the present study may be even more challenging for cartilage repair, the finding that articular cartilage regeneration did not occur in the lacZ control defects suggests that this model system is valid to investigate articular cartilage repair. Defects of similar size have been recently employed to study the effect of overexpression of therapeutic genes on articular cartilage repair $[44,46,47]$.

The mechanisms by which FGF-2 induces an improved articular cartilage repair remain to be elucidated. Previous studies have suggested that FGF-2 mainly acts as a mitogen for mesenchymal cells including fibroblasts [5] and chondrocytes [7,8,27]. FGF-2 also stimulates chemotactic migration of marrow-derived mesenchymal cells [48] into articular cartilage defects [14], resulting in a higher cell density. The enhanced filling at 3 weeks of defects receiving FGF-2 spheres may reflect the beneficial effect of the therapeutic treatment upon cell recruitment, while the increased overall repair at 3 weeks suggests an early stimulating effect on chondrogenesis. The improvement in the cell morphology parameter at 14 weeks suggests a long-term effect of FGF-2 on chondrogenesis. It is possible that overexpression of FGF-2 may also influence the release of other growth or transcription factors [49] or the expression of their receptors [50] in both the transplanted chondrocytes and the surrounding cells of mesenchymal origin [51]. Analysis of type II collagen expression suggests that, after 3 weeks, FGF-2 may selectively increase type II collagen content. In contrast, FGF-2 did not lead to an increase in type I collagen production at both time points.

This gene-based model of localized synthesis of therapeutic factors may serve as a prototype to examine the effects of other potentially therapeutic molecules on articular cartilage repair. In the future, it will be important to assess the mechanisms of sphere resorption and to develop approaches to prolong transgene expression. Likewise, it will be important to test other therapeutic candidates in order to further enhance articular cartilage repair. Finally, the long-term durability of the repair tissue needs to be studied in a larger animal model that resembles more closely a clinically relevant situation.

In summary, the results of the present study demonstrate that localized overexpression of a human FGF-2 gene sequence augments chondrogenesis and enhances articular cartilage repair in vivo, without adverse effects on the synovial membrane. These data might be used to define the effects of genes involved in articular cartilage repair and lead to the development of novel gene-based therapies for human articular cartilage defects.

\section{Acknowledgements}

We thank T. Thurn and E. Kabiljagic for expert technical assistance, M. Seno for the hFGF-2 cDNA, and K. Remberger for helpful discussions. Supported by the Deutsche Forschungsgemeinschaft (DFG MA 2363/1-1, H.M.), the AO ASIF foundation and NIH grant RO1 AR47702 (S.B.T.). 


\section{References}

1. O'Driscoll SW. The healing and regeneration of articular cartilage. J Bone Joint Surg Am 1998; 80: 1795-1812.

2. Hunziker EB. Articular cartilage repair: basic science and clinical progress. A review of the current status and prospects. Osteoarthritis Cartilage 2002; 10: 432-463.

3. Shapiro F, Koide S, Glimcher MJ. Cell origin and differentiation in the repair of full-thickness defects of articular cartilage. $J$ Bone Joint Surg Am 1993; 75: 532-553.

4. Trippel SB. Growth factors as therapeutic agents. Instr Course Lect 1997; 46: 473-476.

5. Gospodarowicz D. Localisation of a fibroblast growth factor and its effect alone and with hydrocortisone on 3T3 cell growth. Nature 1974; 249: 123-127.

6. Burgess WH, Maciag T. The heparin-binding (fibroblast) growth factor family of proteins. Annu Rev Biochem 1989; 58: 575-606.

7. Sah RL, Chen AC, Grodzinsky AJ, et al. Differential effects of bFGF and IGF-I on matrix metabolism in calf and adult bovine cartilage explants. Arch Biochem Biophys 1994; 308: 137-147.

8. Trippel SB. Growth factor actions on articular cartilage. $J$ Rheumatol Suppl 1995; 43: 129-132.

9. Schofield JN, Wolpert L. Effect of TGF-beta 1, TGF-beta 2, and bFGF on chick cartilage and muscle cell differentiation. Exp Cell Res 1990; 191: 144-148.

10. Kato Y. Roles of fibroblast growth factor and transforming growth factor- $\beta$ families in cartilage formation. In Biological Regulation of the Chondrocytes, Adolphe M (ed). CRC Press: Boca Raton, 1992; 141-160.

11. Jentzsch KD, Wellmitz G, Heder G, et al. A bovine brain fraction with fibroblast growth factor activity inducing articular cartilage regeneration in vivo. Acta Biol Med Ger 1980; 39: 967-971.

12. Otsuka Y, Mizuta H, Takagi K, et al. Requirement of fibroblast growth factor signaling for regeneration of epiphyseal morphology in rabbit full-thickness defects of articular cartilage. Dev Growth Differ 1997; 39: 143-156.

13. Fujimoto E, Ochi M, Kato Y, et al. Beneficial effect of basic fibroblast growth factor on the repair of full-thickness defects in rabbit articular cartilage. Arch Orthop Trauma Surg 1999; 119: $139-145$

14. Chuma H, Mizuta H, Kudo S, et al. One day exposure to FGF-2 was sufficient for the regenerative repair of full-thickness defects of articular cartilage in rabbits. Osteoarthritis Cartilage 2004; 12: $834-842$.

15. Mizuta H, Kudo S, Nakamura E, et al. Active proliferation of mesenchymal cells prior to the chondrogenic repair response in rabbit full-thickness defects of articular cartilage. Osteoarthritis Cartilage 2004; 12: 586-596.

16. Yamamoto $\mathrm{T}$, Wakitani $\mathrm{S}$, Imoto $\mathrm{K}$, et al. Fibroblast growth factor-2 promotes the repair of partial thickness defects of articular cartilage in immature rabbits but not in mature rabbits. Osteoarthritis Cartilage 2004; 12: 636-641.

17. Edelman ER, Nugent MA, Karnovsky MJ. Perivascular and intravenous administration of basic fibroblast growth factor: vascular and solid organ deposition. Proc Natl Acad Sci U S A 1993; 90: 1513-1517.

18. Shida J, Jingushi S, Izumi T, et al. Basic fibroblast growth factor stimulates articular cartilage enlargement in young rats in vivo. J Orthop Res 1996; 14: 265-272.

19. Evans CH, Ghivizzani SC, Robbins PD. Orthopaedic gene therapy. Clin Orthop 2004; 429: 316-329.

20. Trippel SB, Ghivizzani SC, Nixon AJ. Gene-based approaches for the repair of articular cartilage. Gene Ther 2004; 11: 351-359.

21. Madry H, Cucchiarini M, Stein U, et al. Sustained transgene expression in cartilage defects in vivo after transplantation of articular chondrocytes modified by lipid-mediated gene transfer in a gel suspension delivery system. J Gene Med 2003; 5: 502-509.

22. Baragi VM, Renkiewicz RR, Qiu L, et al. Transplantation of adenovirally transduced allogeneic chondrocytes into articular cartilage defects in vivo. Osteoarthritis Cartilage 1997; 5: 275-282.

23. Ikeda T, Kubo T, Nakanishi T, et al. Ex vivo gene delivery using an adenovirus vector in treatment for cartilage defects. $J$ Rheumatol 2000; 27: 990-996.

24. Hirschmann F, Verhoeyen E, Wirth D, et al. Vital marking of articular chondrocytes by retroviral infection using green fluorescence protein. Osteoarthritis Cartilage 2002; 10: 109-118.

25. Mierisch CM, Wilson HA, Turner MA, et al. Chondrocyte transplantation into articular cartilage defects with use of calcium alginate: the fate of the cells. $J$ Bone Joint Surg Am 2003; 85A: 1757-1767.

26. Madry H, Cucchiarini M, Terwilliger EF, et al. Recombinant adeno-associated virus vectors efficiently and persistently transduce chondrocytes in normal and osteoarthritic human articular cartilage. Hum Gene Ther 2003; 14: 393-402.

27. Madry H, Emkey G, Zurakowski D, et al. Overexpression of human fibroblast growth factor 2 stimulates cell proliferation in an ex vivo model of articular chondrocyte transplantation. $J$ Gene Med 2004; 6: 238-245.

28. Farndale RW, Buttle DJ, Barrett AJ, et al. Improved quantification of sulphated glycosaminoglycans by use of dimethylmethylene blue. Biochim Biophys Acta 1986; 883: 173-177.

29. Kiernan JA (ed). Histological and Histochemical Methods-Theory and Practice, Butterworth-Heinemann: Oxford, 1999.

30. Nixon AJ, Fortier LA, Williams J, et al. Enhanced repair of extensive articular defects by insulin-like growth factor-I-laden fibrin composites. J Orthop Res 1999; 17: 475-487.

31. Fortier LA, Mohammed HO, Lust G, et al. Insulin-like growth factor-I enhances cell-based repair of articular cartilage. $J$ Bone Joint Surg Br 2002; 84: 276-288.

32. Sellers RS, Peluso D, Morris EA. The effect of recombinant human bone morphogenetic protein-2 (rhBMP-2) on the healing of full-thickness defects of articular cartilage. J Bone Joint Surg Am 1997; 79: 1452-1463.

33. Sellers RS, Zhang R, Glasson SS, et al. Repair of articular cartilage defects one year after treatment with recombinant human bone morphogenetic protein-2 (rhBMP-2). $J$ Bone Joint Surg Am 2000; 82: 151-160.

34. Cuevas P, Burgos J, Baird A. Basic fibroblast growth factor (FGF) promotes cartilage repair in vivo. Biochem Biophys Res Commun 1988; 156: 611-618.

35. Uteza Y, Rouillot JS, Kobetz A, et al. Intravitreous transplantation of encapsulated fibroblasts secreting the human fibroblast growth factor 2 delays photoreceptor cell degeneration in Royal College of Surgeons rats. Proc Natl Acad Sci U S A 1999; 96: 3126-3131.

36. Qu Z, Huang XN, Ahmadi P, et al. Expression of basic fibroblast growth factor in synovial tissue from patients with rheumatoid arthritis and degenerative joint disease. Lab Invest 1995; 73: 339-346.

37. Manabe $\mathrm{N}$, Oda $\mathrm{H}$, Nakamura $\mathrm{K}$, et al. Involvement of fibroblast growth factor-2 in joint destruction of rheumatoid arthritis patients. Rheumatology (Oxford) 1999; 38: 714-720.

38. Lim F, Sun AM. Microencapsulated islets as bioartificial endocrine pancreas. Science 1980; 210: 908-910.

39. Chang PL. Microcapsules as bio-organs for somatic gene therapy. Ann N Y Acad Sci 1997; 831: 461-473.

40. Soon-Shiong $\mathrm{P}$, Heintz RE, Merideth $\mathrm{N}$, et al. Insulin independence in a type 1 diabetic patient after encapsulated islet transplantation. Lancet 1994; 343: 950-961.

41. Bonaventure J, Kadhom N, Cohen-Solal L, et al. Reexpression of cartilage-specific genes by dedifferentiated human articular chondrocytes cultured in alginate beads. Exp Cell Res 1994; 212: 97-104.

42. Hauselmann HJ, Masuda K, Hunziker EB, et al. Adult human chondrocytes cultured in alginate form a matrix similar to native human articular cartilage. Am J Physiol 1996; 271: C742-752.

43. Rokstad AM, Kulseng B, Strand BL, et al. Transplantation of alginate microcapsules with proliferating cells in mice: capsular overgrowth and survival of encapsulated cells of mice and human origin. Ann N Y Acad Sci 2001; 944: 216-225.

44. Cucchiarini M, Madry $\mathrm{H}$, Ma C, et al. Improved tissue repair in articular cartilage defects in vivo by rAAV-mediated overexpression of human fibroblast growth factor 2 . Mol Ther 2005; 12: 229-238. DOI: 10.1016/j.ymthe.2005.03.012.

45. Yokoo N, Saito T, Uesugi M, et al. Repair of articular cartilage defect by autologous transplantation of basic fibroblast growth factor gene-transduced chondrocytes with adeno-associated virus vector. Arthritis Rheum 2005; 52: 164-170.

46. Madry H, Kaul G, Cucchiarini M, et al. Enhanced repair of articular cartilage defects in vivo by transplanted chondrocytes overexpressing insulin-like growth factor I (IGF-I). Gene Ther 2005; 12: 1171-1179. DOI:10.1038/sj.gt.3302515. 
47. Mason JM, Breitbart AS, Barcia M, et al. Cartilage and bone regeneration using gene-enhanced tissue engineering. Clin Orthop 2000; 379: S171-178.

48. Buckley-Sturrock A, Woodward SC, Senior RM, et al. Differential stimulation of collagenase and chemotactic activity in fibroblasts derived from rat wound repair tissue and human skin by growth factors. J Cell Physiol 1989; 138: 70-78.

49. Murakami S, Kan M, McKeehan WL, et al. Up-regulation of the chondrogenic Sox9 gene by fibroblast growth factors is mediated by the mitogen-activated protein kinase pathway. Proc Natl Acad Sci U S A 2000; 97: 1113-1118.
50. Hernandez-Sanchez C, Werner H, Roberts CT Jr, et al. Differential regulation of insulin-like growth factor-I (IGF-I) receptor gene expression by IGF-I and basic fibroblastic growth factor. $J$ Biol Chem 1997; 272: 4663-4670.

51. Schollmann S, Grugel R, Tatjej D, et al. Basic fibroblast growth factor modulates the mitogenic potency of the platelet-derived growth factor (PDGF) isoforms by specific up-regulation of the PDGF alpha receptor in vascular smooth muscle cells. $J$ Biol Chem 1992; 267: 18032-18039. 